\title{
Design of a Low-Energy Electrostatic Storage Ring under Consideration of 3D Fields
}

\author{
Mohamed O. A. El Ghazaly ${ }^{1,2,3}$
}

1. King Abdulaziz City for Science and Technology (KACST), PO Box 6086, Riyadh 11442, Saudi Arabia

2. Institute of Condensed Matter and Nanosciences, Catholic University of Louvain, B-1348 Louvain-laNeuve, Belgium

3. Atomic and Molecular Physics Group, Jet Propulsion Laboratory/Caltech, 4800 Oak Grove Drive, Pasadena CA 91109, USA

A low-energy electrostatic storage ring [1] has been developed, and is currently being built as a new core laboratory for atomic and molecular collisions [2] at the King Abdulaziz City for Science and Technology (KACST), in Riyadh, Saudi Arabia. This ring follows the standard design of the pioneering storage ring ELISA [3] and like it, features a racetrack shaped layout. The lattice of the ring was investigated using the MAD [4], which makes use of transfer matrices in an abrupt boundary model of the field distributions. The SIMION program [5], based on solving Laplace's equation numerically, allows the ions to be tracked in more realistic distributions of the electrostatic fields. SIMION was used to model the lattice and the field distribution, considering non-linear components of the fields, such as fringing fields and aberrations. The design, simulations and construction of such a storage ring are reported in detail in [6]. This paper describes the standard design of low-energy electrostatic storage rings, and gives an overview on the use of SIMION for an in-depth optimization of the design.

Electrostatic storage rings [3] rely on the exclusive use of electrostatic optics and they share the advantages and challenges associated with the use of electric fields in low-energy beam experiments. Principally, an electrostatic ring provides the possibility to store the beam whatever the mass of the ion. This unique feature arises from the inherent property of the electric field, which has a mass-independent bending effect on ions of the same kinetic energy. Indeed, the electric field strength $(E)$ required to bend an ion of charge $q$ and a kinetic energy $T$, with bending radius $\rho$, is proportional to the quantity $T / q \rho$. When expressing the kinetic energy $T$ in terms of the initial acceleration potential $\left(\Delta U_{a c c}\right)$, the electrostatic field rigidity $(\rho E)$ can be written as [1]:

$$
E=\frac{2}{\rho} \Delta U_{a c c}
$$

Expression (1), which describes the strength of the electrostatic bending field, is independent of the mass of the ion, and so there is no mass limit for the storage of molecular ions in an electrostatic ring. Thereby, electrostatic storage rings are a suitable tool for investigating the molecular dynamics of clusters or macromolecular ions. Electrostatic storage rings typically operate at a nominal energy range of $20-50 \mathrm{keV}$. Nevertheless, the use of electrostatic ion-optics with low-energy beams is inherently associated with nonlinear field effects such as aberrations or fringing fields, which can compromise the beam lifetime and limit the acceptance of the ring. In a low-energy storage ring it is rather the interplay of single-turn aberrations over multiple turns that can lead to such drastic effects [7]. These non-linear effects have to be taken into account in the routines commonly used in designing electrostatic storage rings, in order to further improve the performance of the ring. 
The design of a low-deflecting electrostatic storage ring, referred to as the $7^{\circ}$ design, has been studied earlier [8]. In this $7^{\circ}$ design, chromatic and spherical aberrations from a prototypical electrostatic quadrupole have been investigated and the geometry of the main bending optics was optimized with respect to fringing-field extensions according to the theoretical calculation of the effective field boundary [9]. Although the SIMION ion trajectory simulations have demonstrated the ability of an electrostatic ring to store low-energy beams in spite of fringe fields, limitations in acceptance and dynamic aperture of the ring are observed. In the design outlined here [1], the lattice has been optimized under consideration of non-linear effects, using the SIMION code, which accurately computes ion trajectories in realistic distributions of the electrostatic field.

The standard design refers to the ELectrostatic Ion Storage ring, Aarhus (ELISA) [3] designed for atomic and molecular physics. The pioneering storage ring ELISA typifies a single-bend, racetrackshaped ring, the lattice of which relies solely on linear ion optics. It only makes use of the electrostatic field with dipoles to bend the beam and quadrupoles to focus it. The overall circumference of the ring is about $7 \mathrm{~m}$. In this straightforward concept, no optics is used to compensate for nonlinear effects [6]. Therefore, the typical lattice of the standard design consists of a $160^{\circ}$ cylindrical deflector (CD) inserted between two $10^{\circ}$ Parallel Deflecting Plates (PDP) and quadrupole doublets for shaping the beam. The quadrupoles defocus in one transverse direction, while they focus in the other, and so it is used in a double set in order to ensure a net focusing in the transverse plane. Figure 1 shows the prototypical design of shielded pairs of deflecting plates, a cylindrical deflector, and a quadrupole doublet, as used in the standard design.

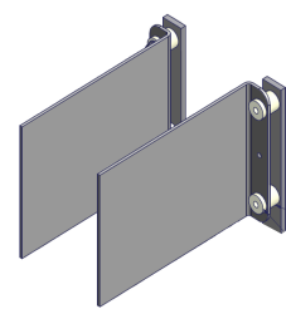

(a)

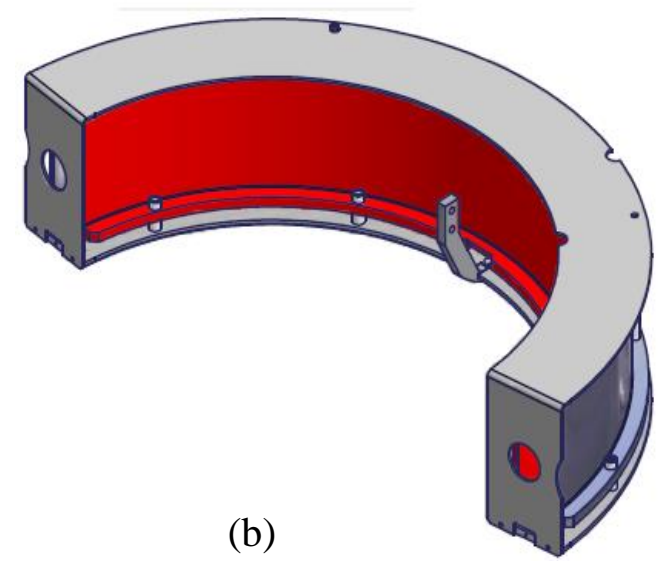

(b)

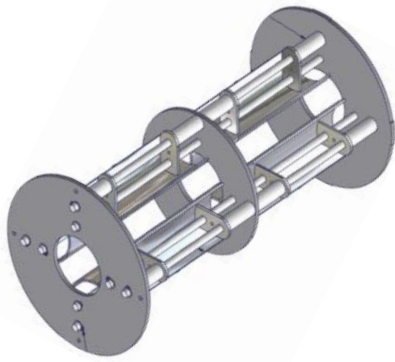

(c)

Figure 1. (a) Parallel Deflecting Plate (PDP), (b) Cylindrical Deflector (CD), and (c) quadrupole doublet.

Most of the existing electrostatic storage rings, whether single or double, feature the standard or ELISAlike design. Other electrostatic storage rings feature a square-shaped or quadrilateral ring [10] in splitbend [11] or $90^{\circ}$ turning quadrupole configurations [12], a smaller deflecting angle in the parallel plates $[8,11]$ of a single-bend racetrack or split-bend, square-shaped layout; or a larger angle in a split-bend, racetrack layout [13]. Small deflection angles reduce aberrations. The standard design involves a limited number of optical elements, which are used to reduce nonlinear field effects such as aberrations and fringing fields. 
The potential generated by the standard ion-optical elements (deflectors and quadrupoles) is welldocumented $[14,15]$. Under the assumption of infinitely long cylinders, the electrostatic potential inside the CD can be determined by using Gauss's theorem. In cylindrical coordinates, the potential at a radius $r$, which is between the two coaxial electrodes with radii $R_{1}$ and $R_{2}$ of the CD, is given by [6]

$$
V(r)=-U_{0}+\frac{2 U_{0}}{\ln \left(R_{2} / R_{1}\right)} \ln \left[\frac{r}{R_{1}}\right],
$$

where $\pm U_{\mathrm{o}}$ is the voltage on the electrodes at $R_{1}$ and $R_{2}$ of the CD. This expression is used in the equations of motion to determine the trajectory of stored ions inside the $\mathrm{CD}$. The equations of motion in the transverse plane $(x, y)$ of ions under this potential are [6]

$$
\begin{aligned}
& \text { (Horizontal) } \frac{d^{2} x}{d s^{2}}+\frac{2}{\rho^{2}} x=\frac{1}{\rho} \frac{\Delta T}{T}, \\
& \text { (Vertical) } \quad \frac{d^{2} y}{d s^{2}}=0 .
\end{aligned}
$$

Ions entering the quadrupole are subject to an electrostatic potential $V(x, y)$

$$
V(x, y)=-\frac{U_{0}}{a_{0}^{2}}\left(x^{2}-y^{2}\right) .
$$

This potential makes ions oscillate back and forth around the nominal trajectory, following the equations:

$$
\begin{array}{ll}
\text { (Horizontal) } & \frac{d^{2} x}{d s^{2}}+k x=0, \\
\text { (Vertical) } & \frac{d^{2} y}{d s^{2}}-k y=0,
\end{array}
$$

with the quadrupole strength $k=q U_{0} / a_{0}^{2} T$, where $a_{o}$ is the inscribed radius of the quadrupole.

Although it is designed by using only linear ion-optics, the field is not linear along the optical axis of the elements (PDP, CD or quads) because of fringing on the edges, as can be seen in the example (Figure 2). Linear transfer matrices, in this case, do not provide an accurate solution of the equations of motion, since they are derived from an abrupt boundary model of the field distributions. This idealization of the field distributions, as commonly done using transfer matrix codes such as MAD, comes at the expense of accuracy and performance of the design. Nevertheless, MAD is commonly used to perform lattice calculations and to determine stable solutions. 


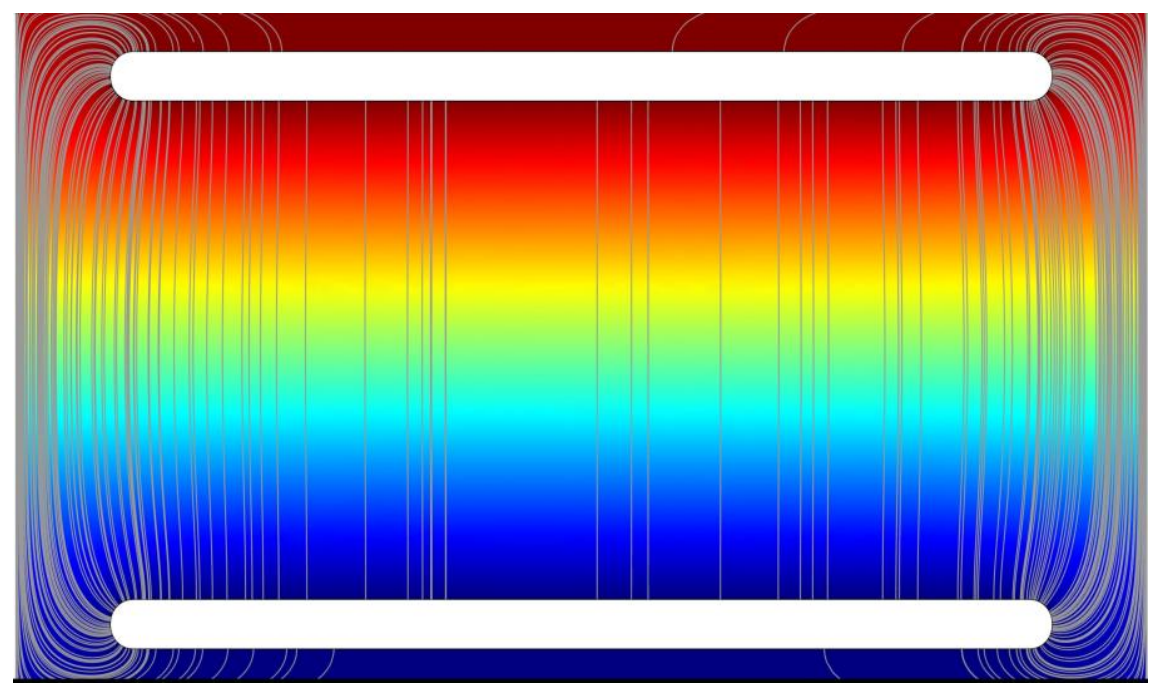

Figure 2. Electric field lines in a parallel deflecting plate (PDP), as calculated by COMSOL [16].

In the absence of space charge, the electrostatic potential $V(s)$ along the nominal trajectory $(s)$ is a solution to Laplace's equation. SIMION 8.0 was used to accurately reproduce the shape of the optical elements and to optimize their design with respect to fringe fields, where SIMION uses the finite difference method to solve Laplace's equation. Alternatively, the code COSY Infinity [17] which uses transfer maps, is used to reproduce realistic field distributions. Ray-tracing simulations were then performed to determine the ion-optical aberrations in the optimized design of optical elements [6]. Furthermore, a layout is designed on the basis of the super periodicity of the lattice and the maximized mirror symmetry. In this way, the ring can be modeled with relatively high grid density (up to 3 grid points $/ \mathrm{mm}$ ). Ion trajectory simulations were then conducted to test the performance of such an optimized design. In this design, large ion beams up to $10 \mathrm{~mm}$ size were stably stored in the ring for hours of turns. Figure 3 shows the ion-beam stored in the ring as modelled by SIMION 8.0. Further in-depth investigations were performed using SIMION and other tracking software to probe the beam stability and storage time of the ring [6].

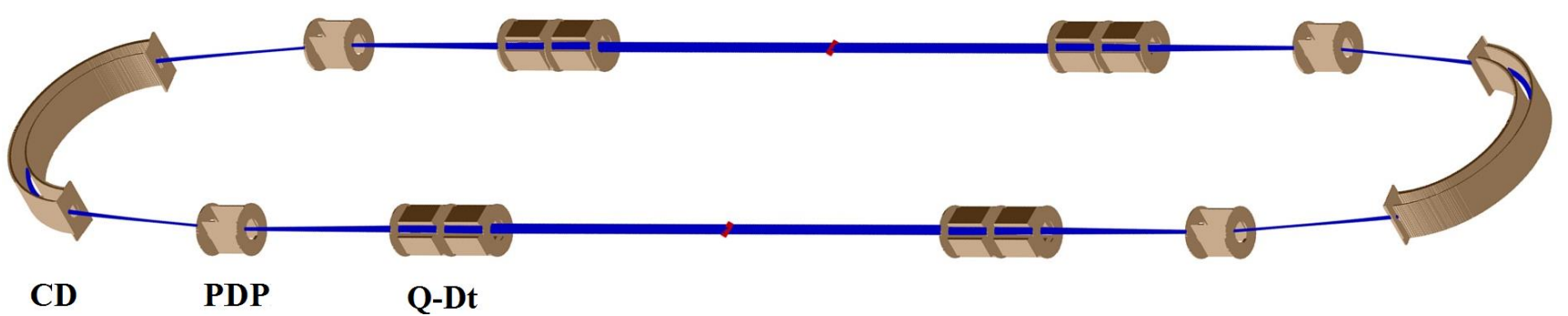

Figure 3. A $10 \mathrm{~mm}$ diameter ion-beam tracked through an electrostatic storage ring designed by SIMION. Here, Q-Dt denotes a quadrupole doublet.

A design of a low energy electrostatic storage ring has been worked out by using SIMION. The design was optimized with regards to the non-linear fields, such as fringing fields and aberrations. These 
simulations are the basis of the design of the low-energy electrostatic storage ring that is currently being assembled. Shown in Figure 4 is a photograph of the vacuum chamber body of the new electrostatic storage ring [18].

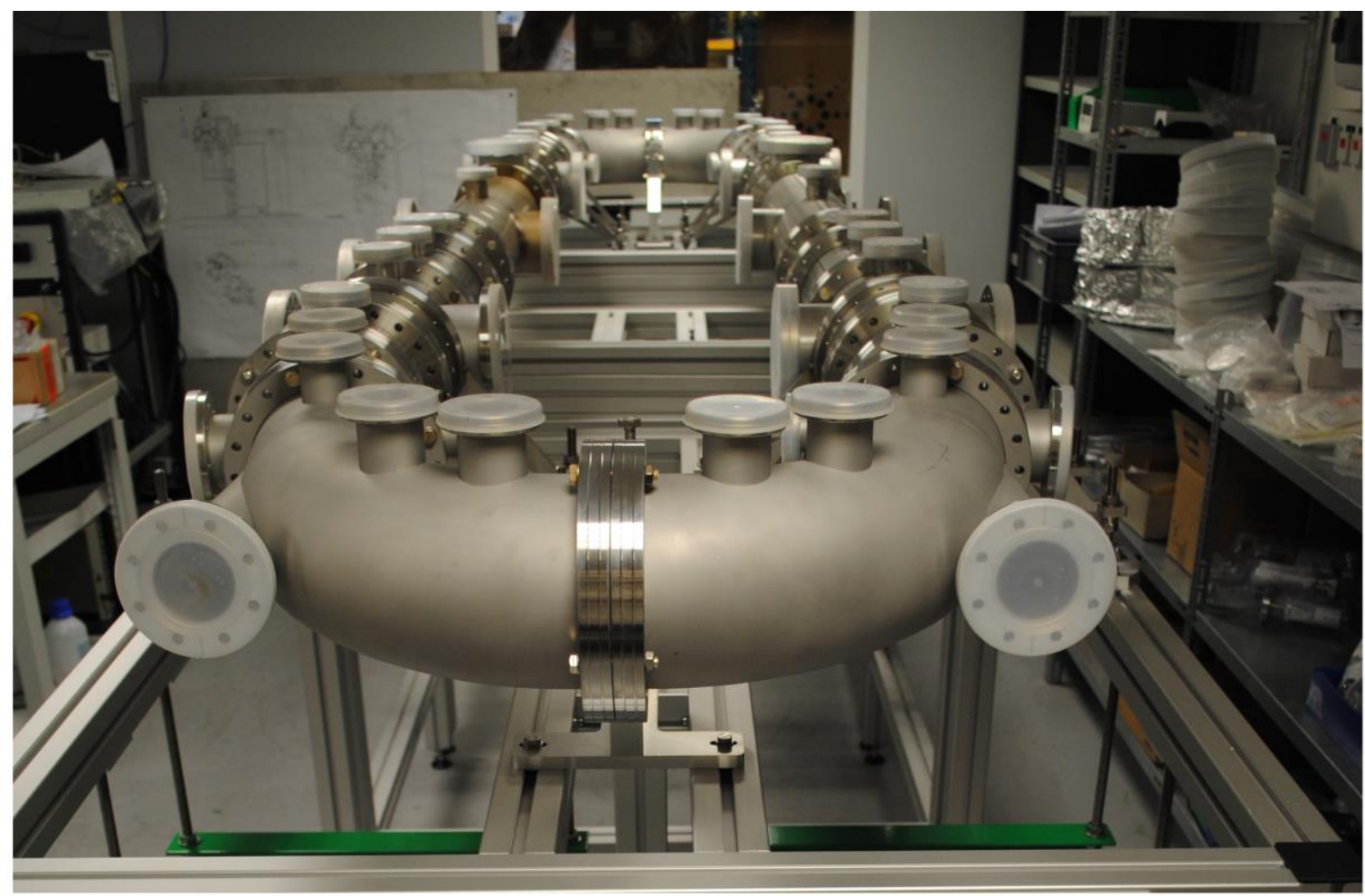

Figure 4. The vacuum chamber body of the electrostatic storage ring under assembly.

References:

[1] MOA El Ghazaly, EPJ Web of Conferences 84 (2015), 05003.

[2] MOA El Ghazaly, $4^{\text {th }}$ International Workshop on Electrostatic Storage Devices-ESD11 (2011) p. 39.

[3] SP Møller, Nucl. Instrum. Meth. A 394 (1997), p. 281.

[4] http://mad.web.cern.ch/mad.

[5] SIMION 3D v8.0, Scientific Instrument Services, Inc., http://www.simion.com.

[6] MOA El Ghazaly et al, under preparation (2015).

[7] M Berz and K Makino, Nucl. Instrum. Meth. A 645 (2011), p. 175.

[8] MOA El Ghazaly et al, Nucl. Instrum. Meth. A 709 (2013), p. 76.

[9] H Wollnik in "Optics of Charged Particles", (Academic Press, Orlando, 1987).

[10] MOA El Ghazaly, XXVIII International Conference on Photonic, Electronic and Atomic Collisions-ICPEAC2013 (2013) 22691.

[11] D Zajfman et al, J. Phys. Conf. Ser. 4 (2005), p. 296 c.

[12] HB Petersen, $5^{\text {th }}$ International Workshop on Electrostatic Storage Devices-ESD13 (2013) p. I22.

[13] K Stiebing et al, Nucl. Instrum. Meth. A 614 (2010), p. 10.

[14] M Ikegami et al, Phys. Rev. Spec. Top. Ac.7 (2004), 120101.

[15] Y Senichev et al, EPAC2000 Proceedings (2000) p.2166. 
[16] MOA El Ghazaly et al, AIP AIP Conf. Proc. 1370 (2011), p. 272.

[17] http://www.bt.pa.msu.edu/index_cosy.htm.

[18] The author gratefully acknowledges the helpful comments and useful suggestions from the anonymous reviewer. The author acknowledges funding from the King Abdulaziz City for Science and Technology (KACST), Grant no. 162-28/PI, principal investigator: MOA El Ghazaly. Thanks to all people who have contributed to this project. In particular, P. Defrance, J. B. Mitchell and A. Chutjian are thanked for valuable discussions related to this work. Engineers at Vinci technologies in Paris are warmly thanked. 